MATHEMATICS OF COMPUTATION

Volume 74, Number 251, Pages 1281-1290

S 0025-5718(04)01707-7

Article electronically published on August 26, 2004

\title{
A FINITE DIMENSIONAL REALIZATION OF THE MOLLIFIER METHOD FOR COMPACT OPERATOR EQUATIONS
}

\author{
M. T. NAIR AND SHINE LAL
}

\begin{abstract}
We introduce and analyze a stable procedure for the approximation of $\left\langle f^{\dagger}, \varphi\right\rangle$ where $f^{\dagger}$ is the least residual norm solution of the minimal norm of the ill-posed equation $A f=g$, with compact operator $A: X \rightarrow Y$ between Hilbert spaces, and $\varphi \in X$ has some smoothness assumption. Our method is based on a finite number of singular values of $A$ and some finite rank operators. Our results are in a more general setting than the one considered by Rieder and Schuster (2000) and Nair and Lal (2003) with special reference to the mollifier method, and it is also applicable under fewer smoothness assumptions on $\varphi$.
\end{abstract}

\section{INTRODUCTION}

Many inverse problems in science and engineering have their mathematical formulation as an operator equation

$$
\text { Af }=g
$$

where $A: X \rightarrow Y$ is a compact operator between Hilbert spaces $X$ and $Y$ (cf. [3, 5, 7, 6, 20]). It is well known that if $A$ is of infinite rank, then the problem of solving the above equation is ill-posed, in the sense that the generalized solution $f^{\dagger}:=A^{\dagger} g$ for $g \in D\left(A^{\dagger}\right):=R(A)+R(A)^{\perp}$ does not depend continuously on the data $g$ (cf. [3, 4, 14]). Here $A^{\dagger}$ denotes the Moore-Penrose generalized inverse of $A$. We may recall that for $g \in D\left(A^{\dagger}\right), f^{\dagger}:=A^{\dagger} g$ is the minimum norm solution of the normalized equation $A^{*} A f^{\dagger}=A^{*} g$. A typical example of a compact operator with infinite rank which often occurs in practical problems is the Fredholm integral equation of the first kind,

$$
\int_{\Omega} k(s, t) f(t) d t=g(s), \quad s \in \Omega,
$$

where $k(\cdot, \cdot)$ is a non-degenerate kernel, so that the corresponding integral operator is a compact operator between suitable function spaces. So, in order to obtain stable approximate solutions for such problems, one has to apply certain regularization procedures. There are many regularization procedures available in the literature ((cf. [3, 4, 5, 7]) and the references therein).

In regularization methods, such as the Tikhonov regularization (cf. 3, 4]) or Lavrentiev regularization (cf. $[13,[19]$ ) or in any of their variants and generalizations

Received by the editor December 10, 2003.

2000 Mathematics Subject Classification. Primary 65J10; Secondary 65R10.

(C)2004 American Mathematical Society 
(cf. [3, 4]), one finds a family of regularized solutions of the form

$$
f_{\eta}^{\delta}:=R_{\eta} g^{\delta}
$$

where $g^{\delta}$ is the available data such that $\left\|g-g^{\delta}\right\| \leq \delta$ for some known error level $\delta>0$, and the parameter $\eta>0$ is chosen depending on $\left(g^{\delta}, \delta\right)$ in such a way that $f_{\eta}^{\delta} \rightarrow f^{\dagger}$ as $\delta \rightarrow 0$. In the above procedures, in obtaining the regularized solutions $f_{\eta}^{\delta}$, one has to solve certain well-posed problems for each data $g^{\delta}$. So, it is desirable to have a procedure which gives regularized approximations for different data without having to solve different problems. Also, usually in practical problems, the space $X$ is a space of functions defined on certain subset $\Omega$ of $\mathbb{R}^{k}$, and one is interested in the value of the solution $f^{\dagger}$ at certain points $x$ in $\Omega$. Having such situations in mind, Louis and Maass [8] considered a new procedure, the so-called mollifier method, for approximating $f^{\dagger}(x)$. In this procedure, one considers a family $\mathcal{E}:=\left\{e_{\gamma}(\cdot, \cdot): \gamma>0\right\}$ of functions on $\Omega \times \Omega$, called mollifiers, such that $e_{\gamma}(x, \cdot)$ is well defined, $e_{\gamma}(x, \cdot) \in X$ for each $x \in \Omega$ and $\left\langle f, e_{\gamma}(x, \cdot)\right\rangle \rightarrow f(x)$ as $\gamma \rightarrow 0$. Moreover, $\mathcal{E}$ is required to have some additional property so that $\left\langle f, e_{\gamma}(x, \cdot)\right\rangle$ can be expressed as $\left\langle g, \psi_{\gamma}(x)\right\rangle$ for some $\psi_{\gamma}(x) \in Y$. By the first requirement $E_{\gamma} f^{\dagger}$ defined by $\left(E_{\gamma} f\right)(x)=\left\langle f, e_{\gamma}(x, \cdot)\right\rangle, x \in \Omega$, can be considered as an approximation of $f^{\dagger}$ for small enough $\gamma$, and by the latter requirement the computation of $E_{\gamma} f^{\dagger}$ is reduced in terms of known quantities.

It has been proved by the authors (cf. [15]) that if the data $g$ and the mollifier $e_{\gamma}(x, \cdot)$ are in the domains of the generalized inverses of $A$ and $A^{*}$, respectively, then

$$
\left\langle A^{\dagger} g, e_{\gamma}(x, \cdot)\right\rangle=\left\langle g, \psi_{\gamma}(x)\right\rangle
$$

where $\psi_{\gamma}(x)=\left(A^{*}\right)^{\dagger} e_{\gamma}(x, \cdot)$. Note that the computation of $\left\langle g, \psi_{\gamma}(x)\right\rangle$ involves the problem of solving an ill-posed equation

$$
A A^{*} \psi_{\gamma}(x)=A e_{\gamma}(x, \cdot)
$$

in the infinite dimensional setting. Thus, the need arises to have a finite dimensional procedure to approximate the quantity $\left\langle g, \psi_{\gamma}(x)\right\rangle$. As we have already pointed out, one may be interested in computing the values of $\left\langle g, \psi_{\gamma}(x)\right\rangle$ for a certain finite number of $x$ 's, say for $x \in\left\{x_{1}, \ldots, x_{\ell}\right\}$. Thus, the problem is to get approximations for $\left\langle g, \psi_{\gamma}\left(x_{i}\right)\right\rangle, i=1, \ldots, \ell$.

Rieder and Schuster (cf. [17, [18) suggested a new method for finding an approximation to $\psi_{\gamma}(x)$ by solving a finite dimensional system. In their analysis, first they consider the case of $e_{\gamma}\left(x_{i}, \cdot\right) \in R\left(A^{*}\right)$ and then the general case $e_{\gamma}\left(x_{i}, \cdot\right) \in X$. But the main theorem on error estimates for the general case $e_{\gamma}\left(x_{i}, \cdot\right) \in X$ involves an assumption which amounts to the assumption $e_{\gamma}\left(x_{i}, \cdot\right) \in R\left(A^{*}\right)$. In [15] the authors suggested a new method in the finite dimensional setting for a bounded linear operator $A$ which is applicable for the case of $e_{\gamma}\left(x_{i}, \cdot\right) \notin R\left(A^{*}\right)$ as well. The purpose of this paper is to consider another procedure for the specific case when $A$ is a compact linear operator. In this case we arrive at better estimates than the ones available in [15]. A particular case of the procedure yields an error bound of the form obtained by Rieder and Schuster (cf. [17, 18]) under certain weaker assumptions.

We shall be carrying out our analysis in a slightly more general setting, in which we are approximating $\left\langle f^{\dagger}, \varphi\right\rangle$ which is the same as $\left\langle g,\left(A^{*}\right)^{\dagger} \varphi\right\rangle$ (cf. [15]) whenever $g$ and $\varphi$ are in the domains of $A^{\dagger}$ and $\left(A^{*}\right)^{\dagger}$, respectively, and $X$ is not necessarily 
a function space. In the usual mollifier method, $\varphi$ could be $e_{\gamma}\left(x_{i}, \cdot\right)$, whenever $X$ is a space of functions on $\Omega$ and $e_{\gamma}(\cdot, \cdot)$ is a mollifier.

\section{BACKGROUND MATERIALS}

In our procedure for obtaining stable approximate solutions for the operator equation (1.1) where $A: X \rightarrow Y$ is a compact operator between the Hilbert spaces $X$ and $Y$, we further require a subspace $Z$ of $Y$ which is a Hilbert space with respect to the norm $\|\cdot\|_{Z}$. Norms on $X$ and $Y$ are denoted by $\|\cdot\|$. For example, if $Y=L^{2}(\Omega)$, then $Z$ may be a Sobolev space.

In the mollifier method, we may have $X$ as a function space defined on some subset $\Omega \subseteq \mathbb{R}^{d}$. Our objective is to find $\langle f, \varphi\rangle$ which is the same as $\left\langle g,\left(A^{*}\right)^{\dagger} \varphi\right\rangle$. Also, the evaluation of the inner product $\left\langle g,\left(A^{*}\right)^{\dagger} \varphi\right\rangle$ may involve the integral. Hence, in order to approximate $\left\langle g,\left(A^{*}\right)^{\dagger} \varphi\right\rangle$, we are going to replace it by an inner product in $\mathbb{C}^{n}$ which is easily computable. For that we require $n$ linear functionals $\psi_{n, 1}, \ldots, \psi_{n, n}$ on $Z$ and $n$ elements $\phi_{n, 1}, \ldots, \phi_{n, n}$ in $Y$ such that the linear operator $\Pi_{n}: Z \rightarrow Y$ defined by

$$
\Pi_{n} v=\sum_{k=0}^{n}\left\langle\psi_{n, k}, v\right\rangle \phi_{n, k}, \quad v \in Z,
$$

has the following properties:

- $\exists$ a sequence $\left(\rho_{n}\right)$ in $[0,1]$ which converges to 0 such that

$$
\left\|\Pi_{n} v-v\right\| \leq \rho_{n}\|v\|_{Z} \quad \forall v \in Z
$$

- $\exists c>0$ such that

$$
\left\|\Pi_{n} v\right\| \leq c\|v\|_{Z} \quad \forall v \in Z .
$$

We shall also make use of the operators $\Psi_{n}: Z \rightarrow \mathbb{C}^{n}$ and $\Phi_{n}: \mathbb{C}^{n} \rightarrow Y$ defined by

$$
\left(\Psi_{n} v\right)_{k}=\left\langle\psi_{n, k}, v\right\rangle:=\psi_{n, k}(v), v \in Z, k=1, \ldots, n,
$$

and

$$
\Phi_{n} x=\sum_{k=1}^{n} x_{k} \phi_{n, k}, x \in \mathbb{C}^{n}
$$

respectively. Here, $x_{k}$ denotes the $k$-th coordinate of $x \in \mathbb{C}^{n}$. With the above notations, we can write $\Pi_{n}$ as

$$
\Pi_{n} v=\Phi_{n} \Psi_{n} v, \quad \forall v \in Z .
$$

For instance, in the 2D-computerized tomography problem (cf. 16, 17), one has to solve the operator equation (1.1) with $A$ as the Radon transform defined by

$$
(A f)(s, \theta)=\int_{-w(s)}^{w(s)} f\left(s \omega+t \omega^{\perp}\right) d t
$$

where $\omega:=(\cos \theta, \sin \theta), \omega^{\perp}:=(-\sin \theta, \cos \theta)$ with $(s, \theta) \in W:=(-1,1) \times(0, \pi)$, and $w(s):=\sqrt{1-s^{2}}$. Here $X=L^{2}(\Omega), Y=L^{2}(W)$ and $Z=H^{m+1}(W)$ for some $m>0$ where $\Omega$ is the unit ball in $\mathbb{R}^{2}$ centered at the origin and $H^{m+1}(W)$ denotes the Sobolev space of order $m+1$ defined on the domain $W$. For $p, q \in \mathbb{N}$, define $\Pi_{p, q}: H^{m+1}(W) \longrightarrow L^{2}(W)$ by

$$
\Pi_{p, q} v=\sum_{i=-q}^{q-1} \sum_{j=0}^{p-1} v\left(s_{i}, \theta_{j}\right) B_{q, i} \otimes B_{p, j}, \quad v \in H^{m+1}(W),
$$


where $B_{p, j}=\chi_{\left[\theta_{j}, \theta_{j+1}\right)}$ and $B_{q, i}=\chi_{\left[s_{i}, s_{i+1}\right)}$ for $s_{i}=\frac{i}{q}$, and $\theta_{j}=j \frac{\pi}{p}, i=$ $-q, \ldots, q-1 ; j=0, \ldots, p-1$. Here, for $u, v \in L^{2}(W), u \otimes v \in L^{2}(W)$ is defined as $(u \otimes v)(s, \theta)=u(s) v(\theta)$ for $(s, \theta) \in W$ and $\chi_{[a, b]}$ denotes the characteristic function on the interval $[a, b]$. Then for $v \in H^{m+1}(W)$ we have (cf. [17]),

$$
\left\|\Pi_{p, q} v-v\right\| \leq h\|v\|_{H^{m+1}(W)}, \quad h=\max \left\{\frac{1}{q}, \frac{\pi}{p}\right\},
$$

and there exists $c_{0}>0$ such that

$$
\left\|\Pi_{p, q} v\right\| \leq c_{0}\|v\|_{H^{m+1}(W)} .
$$

Let $G_{n}$ denotes the Gramm matrix related to the family $\left\{\phi_{n, 1}, \ldots, \phi_{n, n}\right\}$. That is, $G_{n}$ is the $n \times n$ matrix whose ij-th entry, $\left(G_{n}\right)_{i j}$, is given by

$$
\left(G_{n}\right)_{i j}=\left\langle\phi_{n, j}, \phi_{n, i}\right\rangle, i, j=1, \ldots, n .
$$

Using the above definitions, it can be seen (cf. [15]) that

$$
\left\langle\Pi_{n} u, \Pi_{n} v\right\rangle=\left\langle\Psi_{n} u, G_{n} \Psi_{n} v\right\rangle \quad \forall u, v \in Z .
$$

We know that the compact linear operator $A: X \rightarrow Y$ between Hilbert spaces $X$ and $Y$ has the singular value representation as

$$
A f=\sum_{k=1}^{\infty} \sigma_{k}\left\langle f, v_{k}\right\rangle u_{k}, \quad x \in X
$$

where $\left\{v_{k}: k \in \mathbb{N}\right\}$ and $\left\{u_{k}: k \in \mathbb{N}\right\}$ are orthonormal bases for $N(A)^{\perp}$ and $\overline{R(A)}$, respectively. Also, the singular values $\sigma_{k}$ and singular vectors $v_{k}, u_{k}$ are related by

$$
A v_{k}=\sigma_{k} u_{k}, \quad A^{*} u_{k}=\sigma_{k} v_{k}
$$

for $k \in \mathbb{N}$. In the case of the Radon transform defined in (2.6) the singular values and singular vectors are known explicitly (see e.g., [1]; also see [2]).

For the purpose of imposing some conditions on $\varphi$, we require a family $\left\{X_{\nu}\right.$ : $\nu>0\}$ of Hilbert spaces defined as follows: For $\nu>0$, let

$$
X_{\nu}:=\left\{f \in N(A)^{\perp}: \sum_{k=1}^{\infty} \sigma_{k}^{-4 \nu}\left|\left\langle f, v_{k}\right\rangle\right|^{2}<\infty\right\} .
$$

Then it is seen that $X_{\nu}$ is a Hilbert space with inner product and norm defined by

$$
\begin{gathered}
\langle f, v\rangle_{\nu}:=\sum_{k=1}^{\infty} \sigma_{k}^{-4 \nu}\left\langle f, v_{k}\right\rangle\left\langle v_{k}, v\right\rangle, \quad f, v \in X_{\nu}, \\
\|f\|_{\nu}:=\sum_{k=1}^{\infty} \sigma_{k}^{-4 \nu}\left|\left\langle f, v_{k}\right\rangle\right|^{2}, \quad f \in X_{\nu}
\end{gathered}
$$

respectively. We may observe that $X_{\nu} \subseteq X_{\mu}$ whenever $\mu \leq \nu$, and the inclusion map from $X_{\nu}$ to $X_{\mu}$ is continuous. Thus, the family $\left\{X_{\nu}: \nu>0\right\}$ is a Hilbert scale. 


\section{Approximation of $\left\langle f^{\dagger}, \varphi\right\rangle$}

Suppose $\varphi \in X$ and $g \in D\left(A^{\dagger}\right)$. Our main objective is to find an approximation for $\left\langle f^{\dagger}, \varphi\right\rangle$, where $f^{\dagger}:=A^{\dagger} g$. As we have already mentioned, in the mollifier method, we may have $X$ a function space defined on some subset $\Omega \subseteq \mathbb{R}^{d}$ and $\varphi(y):=e_{\gamma}\left(x_{i}, y\right)$ for $x_{i}, y \in \Omega, i=1, \ldots, \ell$.

Throughout the rest of the paper we assume that $\varphi \in X_{\nu}$, where $X_{\nu}$ and its inner product and norm are defined as in (2.11), 2.12), 2.13). We observe that, since $\varphi \in X_{\nu}$, it can be represented as

$$
\varphi=\sum_{k=1}^{\infty} \sigma_{k}^{2 \nu}\left\langle u, v_{k}\right\rangle v_{k}
$$

with $u \in X$ given by

$$
u=\sum_{k=1}^{\infty} \sigma_{k}^{-2 \nu}\left\langle\varphi, v_{k}\right\rangle v_{k} .
$$

We may observe that

$$
\|u\|^{2}=\sum_{k=1}^{\infty} \sigma_{k}^{-4 \nu}\left|\left\langle\varphi, v_{k}\right\rangle\right|^{2}=\|\varphi\|_{\nu}^{2} .
$$

We assume that $u_{k} \in Z$ for all $k \in \mathbb{N}$ and that the $u_{k}$ 's are orthogonal in $Z$. For $\alpha>0$ and $M \in \mathbb{N}$, we define an element $v_{M}^{(\alpha)} \in Y$ as follows:

$$
v_{M}^{(\alpha)}:=\sum_{k=0}^{M-1} \frac{\sigma_{k}}{\sigma_{k}^{2}+\alpha}\left\langle\varphi, v_{k}\right\rangle u_{k} .
$$

Since $u_{k} \in Z$ for all $k \in \mathbb{N}, v_{M}^{(\alpha)}$ is an element in $Z$.

For finding an approximation to $\langle f, \varphi\rangle$, we make use of the following result available in [15]. For the sake of completion of exposition, we are including its proof as well.

Theorem 3.1. Let $\Psi_{n}$ and $G_{n}$ be as in (2.5) and (2.8), respectively, $g \in Z \cap D\left(A^{\dagger}\right)$ and $v \in Z$. Let $f^{\dagger}=A^{\dagger} g$. Then

$$
\left|\left\langle f^{\dagger}, \varphi\right\rangle-\left\langle\Psi_{n} g, G_{n} \Psi_{n} v\right\rangle\right| \leq c_{1}\left\{\rho_{n}\|v\|_{Z}+\left\|A^{*} v-\varphi\right\|\right\},
$$

where $c_{1} \geq \max \left\{\left(c\|g\|_{Z}+\|g\|\right),\left\|f^{\dagger}\right\|\right\}$ and $c>0$ is as in (2.4).

Proof. Let $\Pi_{n}$ be as in (2.2). Then by (2.9) we have

$$
\left\langle f^{\dagger}, \varphi\right\rangle-\left\langle\Psi_{n} g, G_{n} \Psi_{n} v\right\rangle=\left\langle f^{\dagger}, \varphi\right\rangle-\left\langle\Pi_{n} g, \Pi_{n} v\right\rangle .
$$

Hence

$$
\begin{aligned}
\left|\left\langle f^{\dagger}, \varphi\right\rangle-\left\langle\Psi_{n} g, G_{n} \Psi_{n} v\right\rangle\right| & =\left|\left\langle f^{\dagger}, \varphi\right\rangle-\left\langle\Pi_{n} g, \Pi_{n} v\right\rangle\right| \\
& \leq\left|\left\langle f^{\dagger}, \varphi\right\rangle-\left\langle f^{\dagger}, A^{*} v\right\rangle\right|+\left|\left\langle f^{\dagger}, A^{*} v\right\rangle-\left\langle\Pi_{n} g, \Pi_{n} v\right\rangle\right| \\
& \leq\left\|f^{\dagger}\right\|\left\|A^{*} v-\varphi\right\|+\left|\left\langle f^{\dagger}, A^{*} v\right\rangle-\left\langle\Pi_{n} g, \Pi_{n} v\right\rangle\right| .
\end{aligned}
$$

Since $f^{\dagger}=A^{\dagger} g$,

$$
\left\langle f^{\dagger}, A^{*} v\right\rangle=\left\langle A f^{\dagger}, v\right\rangle=\langle P g, v\rangle=\langle g, P v\rangle=\langle g, v\rangle
$$


where $P: Y \longrightarrow Y$ is the orthogonal projections onto $\overline{R(A)}$. Using the above relation, we get

$$
\left|\left\langle f^{\dagger}, \varphi\right\rangle-\left\langle\Psi_{n} g, G_{n} \Psi_{n} v\right\rangle\right| \leq\left\|f^{\dagger}\right\|\left\|A^{*} v-\varphi\right\|+\left|\langle g, v\rangle-\left\langle\Pi_{n} g, \Pi_{n} v\right\rangle\right| .
$$

Using the relations (2.3) and (2.4), we have

$$
\begin{aligned}
\left|\langle g, v\rangle-\left\langle\Pi_{n} g, \Pi_{n} v\right\rangle\right| & \leq\left|\langle g, v\rangle-\left\langle g, \Pi_{n} v\right\rangle\right|+\left|\left\langle g, \Pi_{n} v\right\rangle-\left\langle\Pi_{n} g, \Pi_{n} v\right\rangle\right| \\
& \leq\|g\|\left\|\Pi_{n} v-v\right\|+\left\|\Pi_{n} g-g\right\|\left\|\Pi_{n} v\right\| \\
& \leq \rho_{n}\|g\|\|v\|_{Z}+c \rho_{n}\|g\|_{Z}\|v\|_{Z} \\
& =\left(c\|g\|_{Z}+\|g\|\right) \rho_{n}\|v\|_{Z}
\end{aligned}
$$

Thus,

$$
\left|\left\langle f^{\dagger}, \varphi\right\rangle-\left\langle\Psi_{n} g, G_{n} \Psi_{n} v\right\rangle\right| \leq c_{1}\left\{\rho_{n}\|v\|_{Z}+\left\|A^{*} v-\varphi\right\|\right\},
$$

where $c_{1} \geq \max \left\{\left(c\|g\|_{Z}+\|g\|\right),\left\|f^{\dagger}\right\|\right\}$. This completes the proof.

Now, we derive estimates for $\left\|v_{M}^{(\alpha)}\right\|_{Z}$ and $\left\|A^{*} v_{M}^{(\alpha)}-\varphi\right\|$. We do this in the next two lemmas.

Lemma 3.2. Let $v_{M}^{(\alpha)}$ be as in (3.15). Suppose $\varphi \in X_{\nu}$ for some $\nu \in(0,1]$ and $u \in X$ as in (3.14). Then

$$
\left\|A^{*} v_{M}^{(\alpha)}-\varphi\right\| \leq \sqrt{\alpha^{2 \nu}+\sigma_{M}^{4 \nu}}\|u\| .
$$

Proof. Let $\varphi \in X$. From (3.15) and (2.10), we have

$$
A^{*} v_{M}^{(\alpha)}=\sum_{k=0}^{M-1} \frac{\sigma_{k}^{2}}{\sigma_{k}^{2}+\alpha}\left\langle\varphi, v_{k}\right\rangle v_{k} .
$$

Since $\varphi \in X_{\nu}, 0<\nu \leq 1$, with $\varphi=\sum_{k=0}^{\infty} \sigma_{k}^{2 \nu}\left\langle u, v_{k}\right\rangle v_{k}$ for $u \in X$ as in (3.14), we have

$$
\begin{aligned}
A^{*} v_{M}^{(\alpha)}-\varphi & =\sum_{k=0}^{M-1} \frac{\sigma_{k}^{2+2 \nu}}{\sigma_{k}^{2}+\alpha}\left\langle u, v_{k}\right\rangle v_{k}-\sum_{k=0}^{\infty} \sigma_{k}^{2 \nu}\left\langle u, v_{k}\right\rangle v_{k} \\
& =\sum_{k=0}^{M-1} \frac{-\alpha \sigma_{k}^{2 \nu}}{\sigma_{k}^{2}+\alpha}\left\langle u, v_{k}\right\rangle v_{k}-\sum_{k=M}^{\infty} \sigma_{k}^{2 \nu}\left\langle u, v_{k}\right\rangle v_{k}
\end{aligned}
$$

so that

$$
\begin{aligned}
\left\|A^{*} v_{M}^{(\alpha)}-\varphi\right\|^{2} & =\sum_{k=0}^{M-1}\left(\frac{\alpha \sigma_{k}^{2 \nu}}{\sigma_{k}^{2}+\alpha}\right)^{2}\left|\left\langle u, v_{k}\right\rangle\right|^{2}+\sum_{k=M}^{\infty} \sigma_{k}^{4 \nu}\left|\left\langle u, v_{k}\right\rangle\right|^{2} \\
& \leq \sum_{k=0}^{M-1}\left(\frac{\alpha \sigma_{k}^{2 \nu}}{\sigma_{k}^{2}+\alpha}\right)^{2}\left|\left\langle u, v_{k}\right\rangle\right|^{2}+\sigma_{M}^{4 \nu}\|u\|^{2}
\end{aligned}
$$

We observe that

$$
\begin{aligned}
\sum_{k=0}^{M-1}\left(\frac{\alpha \sigma_{k}^{2 \nu}}{\sigma_{k}^{2}+\alpha}\right)^{2}\left|\left\langle u, v_{k}\right\rangle\right|^{2} & =\alpha^{2 \nu} \sum_{k=0}^{M-1}\left(\frac{\left(\frac{\sigma_{k}^{2}}{\alpha}\right)^{\nu}}{\frac{\sigma_{k}^{2}}{\alpha}+1}\right)^{2}\left|\left\langle u, v_{k}\right\rangle\right|^{2} \\
& \leq \alpha^{2 \nu}\|u\|^{2} .
\end{aligned}
$$

Hence, from (3.18) it follows that

$$
\left\|A^{*} v_{M}^{(\alpha)}-\varphi\right\|^{2} \leq \alpha^{2 \nu}\|u\|^{2}+\sigma_{M}^{4 \nu}\|u\|^{2}=\left(\alpha^{2 \nu}+\sigma_{M}^{4 \nu}\right)\|u\|^{2} .
$$


This completes the proof.

The above lemma shows that if $M$ is such that $\sigma_{M}^{2} \leq \alpha$, then

$$
\left\|A^{*} v_{M}^{(\alpha)}-\varphi\right\| \leq \sqrt{2} \alpha^{\nu}\|u\| .
$$

Lemma 3.3. Let $v_{M}^{(\alpha)}$ be as in (3.15). Assume that $\left\|u_{k}\right\|_{Z} \leq \sigma_{k}^{-\beta}$ for all $k \in \mathbb{N}$ for some $\beta$ with $0 \leq \beta \leq 1, \varphi \in X_{\nu}$ with $0<\nu \leq \frac{\beta+1}{2}$, and $u \in X$ as in (3.14). Then

$$
\left\|v_{M}^{(\alpha)}\right\|_{Z} \leq \alpha^{\nu-\frac{\beta+1}{2}}\|u\| \text {. }
$$

Proof. Since $\varphi \in X_{\nu}$ with $\varphi=\sum_{k=0}^{\infty} \sigma_{k}^{2 \nu}\left\langle u, v_{k}\right\rangle v_{k}$ for $u \in X$ as in (3.14), we have

$$
v_{M}^{(\alpha)}=\sum_{k=0}^{M-1} \frac{\sigma_{k}}{\sigma_{k}^{2}+\alpha}\left\langle\varphi, v_{k}\right\rangle u_{k}=\sum_{k=0}^{M-1} \frac{\sigma_{k}^{2 \nu+1}}{\sigma_{k}^{2}+\alpha}\left\langle u, v_{k}\right\rangle u_{k} .
$$

Since the $u_{k}$ 's are orthogonal in $Z$ and $\left\|u_{k}\right\|_{Z} \leq \sigma_{k}^{-\beta}$ for all $k$,

$$
\begin{aligned}
\left\|v_{M}^{(\alpha)}\right\|_{Z}^{2} & =\sum_{k=0}^{M-1}\left(\frac{\sigma_{k}^{2 \nu+1}}{\sigma_{k}^{2}+\alpha}\right)^{2}\left|\left\langle u, v_{k}\right\rangle\right|^{2}\left\|u_{k}\right\|_{Z}^{2} \\
& \leq \sum_{k=0}^{M-1}\left(\frac{\sigma_{k}^{2 \nu+1}}{\sigma_{k}^{2}+\alpha}\right)^{2}\left|\left\langle u, v_{k}\right\rangle\right|^{2} \sigma_{k}^{-2 \beta} \\
& =\sum_{k=0}^{M-1}\left(\frac{\sigma_{k}^{2\left(\nu-\frac{\beta-1}{2}\right)}}{\sigma_{k}^{2}+\alpha}\right)^{2}\left|\left\langle u, v_{k}\right\rangle\right|^{2} \\
& =\left(\alpha^{\nu-\frac{\beta+1}{2}}\right)^{2} \sum_{k=0}^{M-1}\left(\frac{\left(\frac{\sigma_{k}^{2}}{\alpha}\right)^{\nu-\frac{\beta-1}{2}}}{\frac{\sigma_{k}^{2}}{\alpha}+1}\right)^{2}\left|\left\langle u, v_{k}\right\rangle\right|^{2} .
\end{aligned}
$$

Since $0 \leq \beta \leq 1$ and $0<\nu \leq \frac{\beta+1}{2}$, we have $0<\nu-\frac{\beta-1}{2} \leq 1$ so that

$$
\begin{aligned}
\left\|v_{M}^{(\alpha)}\right\|_{Z}^{2} & =\left(\alpha^{\nu-\frac{\beta+1}{2}}\right)^{2} \sum_{k=0}^{M-1}\left|\left\langle u, v_{k}\right\rangle\right|^{2} \\
& \leq\left(\alpha^{\nu-\frac{\beta+1}{2}}\right)^{2}\|u\|^{2} .
\end{aligned}
$$

Thus

$$
\left\|v_{M}^{(\alpha)}\right\|_{Z} \leq \alpha^{\nu-\frac{\beta+1}{2}}\|u\|
$$

Now we are in a position to find an approximation for $\langle f, \varphi\rangle$.

Theorem 3.4. Let $\Psi_{n}$ and $G_{n}$ be as in (2.5) and (2.8), respectively, and $g \in$ $Z \cap D\left(A^{\dagger}\right)$. Let $f^{\dagger}=A^{\dagger} g$. Assume that $\left\|u_{k}\right\|_{Z} \leq \sigma_{k}^{-\beta}$ for all $k \in \mathbb{N}$ for some $\beta$ with $0 \leq \beta \leq 1$, and $\varphi \in X_{\nu}$ with $0<\nu \leq \frac{\beta+1}{2}$, and $u \in X$ as in (3.14). Then

$$
\left|\left\langle f^{\dagger}, \varphi\right\rangle-\left\langle\Psi_{n} g, G_{n} \Psi_{n} v_{M}^{(\alpha)}\right\rangle\right| \leq c_{1}\left\{\rho_{n} \alpha^{\nu-\frac{\beta+1}{2}}+\alpha^{\nu}+\sigma_{M}^{2 \nu}\right\}\|u\|
$$

where $c_{1}>0$ is as in (3.16) and $c>0$ is as in (2.4). In particular, we have the following:

(a) If $\alpha=c_{0} \rho_{n}^{\frac{2}{\beta+1}}$ for some $c_{0}>0$, then

$$
\left|\left\langle f^{\dagger}, \varphi\right\rangle-\left\langle\Psi_{n} g, G_{n} \Psi_{n} v_{M}^{(\alpha)}\right\rangle\right| \leq c_{1}\|u\|\left(\rho_{n}^{\frac{2 \nu}{\beta+1}}+\sigma_{M}^{2 \nu}\right) .
$$


(b) If $\alpha=c_{0} \rho_{n}^{\frac{2}{\beta+1}}$ for some $c_{0}>0$ and $M$ is such that $\sigma_{M}^{2} \leq \rho_{n}^{\frac{2}{\beta+1}}$, then

$$
\left|\left\langle f^{\dagger}, \varphi\right\rangle-\left\langle\Psi_{n} g, G_{n} \Psi_{n} v_{M}^{(\alpha)}\right\rangle\right|=\mathcal{O}\left(\rho_{n}^{\frac{2 \nu}{\beta+1}}\right) .
$$

Proof. Let $v_{M}^{(\alpha)}$ be as in (3.15). Using the relations (3.17) and (3.19), we have

$$
\left\|A^{*} v_{M}^{(\alpha)}-\varphi\right\| \leq \sqrt{\alpha^{2 \nu}+\sigma_{M}^{2 \nu}}\|u\|
$$

and

$$
\left\|v_{M}^{(\alpha)}\right\|_{Z} \leq \alpha^{\nu-\frac{\beta+1}{2}}\|u\| .
$$

Then by Theorem 3.1 we have

$$
\begin{aligned}
\left|\left\langle f^{\dagger}, \varphi\right\rangle-\left\langle\Psi_{n} g, G_{n} \Psi_{n} v_{M}^{(\alpha)}\right\rangle\right| & \leq c_{1}\left\{\rho_{n}\left\|v_{M}^{(\alpha)}\right\|_{Z}+\left\|A^{*} v_{M}^{(\alpha)}-\varphi\right\|\right\} \\
& \leq c_{1}\left\{\rho_{n} \alpha^{\nu-\frac{\beta+1}{2}}+\sqrt{\alpha^{2 \nu}+\sigma_{M}^{4 \nu}}\right\}\|u\| \\
& \leq c_{1}\left\{\rho_{n} \alpha^{\nu-\frac{\beta+1}{2}}+\alpha^{\nu}+\sigma_{M}^{2 \nu}\right\}\|u\|
\end{aligned}
$$

where $c_{1} \geq \max \left\{\left(c\|g\|_{Z}+\|g\|\right),\left\|f^{\dagger}\right\|\right\}$ and $c>0$ is as in (2.4).

The particular case follow from (3.20).

If $\varphi \in X_{\nu}$ for some $\nu \geq \frac{\beta+1}{2}$, then it is obvious that $\varphi \in X_{\frac{\beta+1}{2}}$. Hence the following corollary is an immediate consequence of the above theorem.

Corollary 3.5. Let $v_{M}^{(\alpha)}$ be as in (3.15). Assume that $\varphi \in X_{\nu}$ with $0<\nu \leq \frac{\beta+1}{2}$, and $u \in X$ as in (3.14). If $\sigma_{M}^{2} \leq \rho_{n}^{\frac{2}{\beta+1}}$ and $\alpha \leq \rho_{n}^{\frac{2}{\beta+1}}$, then

$$
\left|\left\langle f^{\dagger}, \varphi\right\rangle-\left\langle\Psi_{n} g, G_{n} \Psi_{n} v_{M}^{(\alpha)}\right\rangle\right|=\mathcal{O}\left(\rho_{n}\right) .
$$

\section{Concluding Remarks}

Remark 1. We observe that the method of this paper is based on the singular value representation of the compact linear operator $A$, and it is applicable only when the singular elements $\sigma_{k}, v_{k}, u_{k}$ are known for $k=1, \ldots, M$ for some large $M \in \mathbb{N}$. It is to be remarked that in computerized tomography problems, where $A$ is the Radon transform in suitable spaces, it is often the case that the singular elements of $A$ are known explicitly (see e.g., [1]; also see [2]).

Remark 2. In [15] the authors considered general bounded operators $A$ and obtained an approximation for $\left\langle f^{\dagger}, \varphi\right\rangle$ in the form $\left\langle\Psi_{n} g, G_{n} \Psi_{n} v_{\alpha, n}\right\rangle$ with corresponding estimation

$$
\left|\left\langle f^{\dagger}, \varphi\right\rangle-\left\langle\Psi_{n} g, G_{n} \Psi_{n} v_{\alpha, n}\right\rangle\right|=\mathcal{O}\left(\rho_{n}^{\frac{\nu}{2}}\right) .
$$

We may observe that the error estimate (3.21) is better than the above estimate in [15] since $\frac{\nu}{2} \leq \frac{2 \nu}{\beta+1}$ for $0 \leq \beta \leq 1$.

Remark 3. An estimate of the form

$$
\left|\left\langle f^{\dagger}, \varphi\right\rangle-\left\langle\Psi_{n} g, G_{n} \Psi_{n} v_{M}^{(\alpha)}\right\rangle\right|=\mathcal{O}\left(\rho_{n}\right)
$$

(see Corollary 3.5) has also been obtained by Rieder and Schuster [17] with an element $\omega_{M}$ in place of $v_{M}^{(\alpha)}$, where

$$
\omega_{M}:=\sum_{k=0}^{M-1} \sigma_{k}^{-1}\left\langle\varphi, v_{k}\right\rangle u_{k}
$$


which is the $M$-th truncated form of the singular value decomposition of $\left(A^{*}\right)^{\dagger} \varphi$. But, for obtaining such a result, they required $\nu$ to satisfy $\nu>\frac{\beta}{2}+\frac{1}{2}+\frac{1}{4 \eta}$ for some $\eta>0$. Clearly, this assumption of Rieder and Schuster [17] is stronger than our assumption, namely, $\nu \geq \frac{\beta+1}{2}$.

Remark 4. Suppose we make a specific choice of $Z$ as

$$
Y_{\beta}:=\left\{y \in Y: \sum_{k=0}^{\infty} \sigma_{k}^{-2 \beta}\left|\left\langle y, u_{k}\right\rangle\right|^{2}<\infty\right\}
$$

for $0 \leq \beta \leq 1$ with $Y_{0}=N\left(A^{*}\right)^{\perp}$. We observe that $R(A) \subset Y_{\beta}$ for $0 \leq \beta \leq 1$. On $Y_{\beta}$, we define an inner product $\langle\cdot, \cdot\rangle_{\beta}$ as

$$
\langle y, u\rangle_{\beta}:=\sum_{k=0}^{\infty} \sigma_{k}^{-2 \beta}\left\langle y, u_{k}\right\rangle\left\langle u_{k}, u\right\rangle, \quad y, u \in Y_{\beta} .
$$

With this inner product, $Y_{\beta}$ is a Hilbert space and the corresponding norm is given by

$$
\|y\|_{\beta}=\sum_{k=0}^{\infty} \sigma_{k}^{-2 \beta}\left|\left\langle y, u_{k}\right\rangle\right|^{2}, \quad y \in Y_{\beta} .
$$

We note that $u_{k} \in Y_{\beta}$ for all $k \in \mathbb{N}$ and $\left\{\sigma_{k}^{\beta} u_{k}: k \in \mathbb{N}\right\}$ is an orthonormal basis for $Y_{\beta}$ with respect to the norm $\|\cdot\|_{\beta}$. Thus we can apply Theorem 3.4. With this choice of $Z$ as $Y_{\beta}$, the error estimate (3.21) is the same as that in the Nair and Lal paper (15], Theorem 3.7). However, the conditions under which that result in [15] holds is stronger. In fact, in [15], it is required that $\beta / 2 \leq \nu \leq(\beta+1) / 2$ whereas the estimate (3.21) is valid for more values of $\nu$, namely, $0 \leq \nu \leq(\beta+1) / 2$.

\section{ACKNOWLEDGMENT}

Shine Lal gratefully acknowledges the financial support received from the Council of Scientific and Industrial Research (CSIR), Government of India.

\section{REFERENCES}

[1] M.E. Davison, A singular value decomposition for the Radon transform in $n$-dimensional Euclidian space, Numer. Funct. Anal. Optim., 3 (1981), 321-340. MR83c:65274

[2] A. Caponnetto and M. Bertero, Tomography with a finite set of projections, Inverse problems, 13 (1997), 1191-1205. MR98j:44001

[3] H.W. Engl, M. Hanke and A. Neubauer, Regularization of Inverse Problems, Kluwer, Dordrect, 1996. MR97k:65145

[4] C.W. Groetsch, The Theory of Tikhonov Regularization for Fredholm Equations of the First Kind, Pitman Publishing, Boston, London, Melbourne, 1984. MR85k:45020

[5] B. Hofmann, Regularization of Applied Inverse and Ill-Posed Problems, Leipzig: Teubner, 1986. MR88i:65001

[6] M. M. Lavrentiev, Some Improperly Posed Problems of Mathematical Physics, Springer, New York, 1967.

[7] A.K. Louis, Inverse und schlecht gestellte Probleme, Teubner, Stuttgart, 1989. MR.90g:65075

[8] A.K. Louis and P. Maass, A mollified method for linear operator equation of first kind, Inverse problems, 6 (1990), 427-440. MR91g:65130

[9] A.K. Louis, Approximate inverse for linear and some nonlinear problems, Inverse problems, 12 (1996), 175-190. MR96m:65063

[10] A.K. Louis, Application of the approximate inverse to 3D X-ray CT and Ultrasound Tomography, Inverse problems in medical imaging and nondestructive testing; Springer-Verlag Wien, New York. Eds: H.W. Engl, A.K. Louis and W. Rendell (1997), 120-133. MR99c:65113 
[11] A.K. Louis, A unified approach to regularisation methods for linear ill-posed problems, Inverse problems, 15 (1999), 489-498. MR2000b:65111.

[12] A.K. Louis, P.Jonas, Approximate inverse for one dimensional inverse heat conduction problem, Inverse problems, 16 (2000), 175-185. MR2000k:35290

[13] M.T. Nair, An iterated version of Lavrentiev's method for ill-posed equations with approximately specified data, J. Inverse and Ill-Posed Problems, 8(2) (2000), 193-204. MR 2001f:65072

[14] M.T. Nair, Functional Analysis: A First Course, Prentice-Hall of India, New Delhi, 2002.

[15] M.T. Nair and Shine Lal, Finite Dimensional Realization of Mollifier Method: A New Stable Approach, J. Inverse and Ill-Posed Problems 12(5) (2004), 1-7.

[16] F. Natterer, The Mathematics of Computerized Tomography, Wiley, Chichester, UK, 1986. MR: $88 \mathrm{~m}: 44008$

[17] A. Rieder and T. Schuster, The Approximate Inverse in Action with an Application to Computerized Tomography, SIAM.J. Numer. Anal., 37 (2000), 1909-1929. MR2001a:65072

[18] A. Rieder and T. Schuster, The Approximate Inverse in Action II: Convergence and Stability, Math. Comput., 72 (2003), 1399-1415. MR2004c:65059

[19] U. Tautenhahn, On the method of Lavrentiev regularization for nonlinear ill-posed problems, Inverse Problems 18 (2002), 191-207. MR2002m:47079

[20] A. N. Tikhonov and V.Y. Arsenin, Solution of Ill-Posed Problems, Wiley, New York, 1977. MR56:13604

Department of Mathematics, Indian Institute of Technology Madras, Chennai 600 036, INDIA

E-mail address: mtnair@iitm.ac.in

Department of Mathematics, Indian Institute of Technology Madras, Chennai 600 036, INDIA

E-mail address: lalshine@hotmail.com 